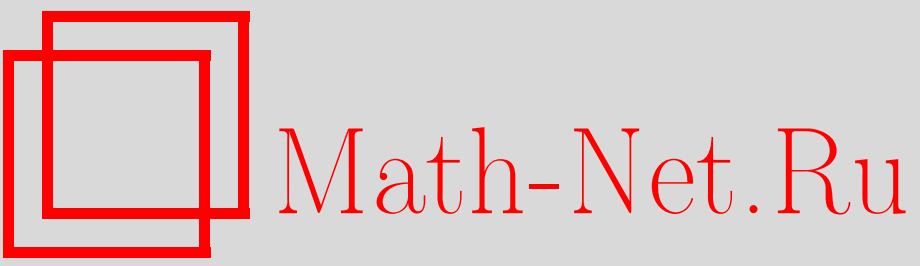

М. Йосвиг, Группа проективностей и раскраска фасет простого многогранника, УМH, 2001, том 56, выпуск 3, 171-172

DOI: https://doi.org/10.4213/rm407

Использование Общероссийского математического портала Math-Net.Ru подразумевает, что вы прочитали и согласны с пользовательским соглашением http://www . mathnet.ru/rus/agreement

Параметры загрузки:

IP: 3.95 .254 .165

26 апреля 2023 г., 17:19:24 


\title{
ГРУППА ПРОЕКТИВНОСТЕЙ И РАСКРАСКА ФАСЕТ ПРОСТОГО МНОГОГРАННИКА
}

\author{
М. Йосвиг
}

В работе [1] М. Дэвисом и Т. Янушкевичем было построено $(n+d)$-мерное гладкое многообразие $\mathscr{Z}_{P}$, определяемое $d$-мерным простым выпуклым многогранником $P$ с $n$ фасетами. Свойства этого многообразия играют решающую роль в исследовании ряда задач из области квазиторических многообразий, см. обзор В. М. Бухштабера и Т. Е. Панова [2]. В работе [3] дано определение хроматического числа $\chi(P)$ простого многогранника $P$ как минимального количества цветов, в которое можно покрасить фасеты многогранника так, чтобы смежные фасеты имели различные цвета. Очевидно, $\chi(P) \geqslant d$ при $\operatorname{dim} P=d$. По любой правильной раскраске многогранника опять же можно построить многообразие, при этом болшшой интерес эта конструкция представляет в случае $\chi(P)=d$. В [3] исследовались многообразия, возникающие при $\chi(P)=d=3$, при этом существенным образом использовался известньй критерий существования правилной трехцветной раскраски фасет простого выпуклого трехмерного многогранника ([3; теорема 1]). В нашей заметке мы покажем, как этот критерий может быть обобщен для многогранника произвольной размерности.

Назовем многогранник четным, если каждая его двумерная грань имеет четное число вершин. Заметим, что каждый зонотоп является четным многогранником, но обратное неверно. Как источник ссылок по теории выпуклых многогранников, можно рекомендовать книгу Г. Циглеpa [4]. Назовем графом многогранника $P$ графф, образованный вершинами и ребрами $P$. Наш основной резуштат состоит в следующем.

ТеОРема 1. Пусть $P$ - простой выпуклый $d$-мерный многогранник. Тогда следующие три условия әквивалентны.

1. Многогранник $P$ четен.

2. $\chi(P)=d$.

3. Граф многогранника $P$ двудолен.

Мы вводим новый комбинаторный инвариант конечномерного симплициального комплекса, группу проективностей, которая затем исследуется в частном случае, когда комплекс является симплициальным многообразием. Напомним, что вьпукльй $d$-мерный многогранник $P$ называется простылм, если каждая его вершина содержится ровно в $d$ фасетах. Это равносильно тому, что граница $\partial P^{*}$ двойственного многогранника $P^{*}$ является симплициальной сферой. Тем самым, резултаты, полученные для симплициальных многообразий, мы можем применять к простым многогранникам. Подробнее о симплициалњных комплексах и симплициалшных многообразиях см. в книге Дж. Манкрса [5].

Пусть $\Delta$ - конечномерньй (абстрактньй) симплициальньй комплекс на множестве вершин $V$. По определению, фасета - это грань максимальной размерности комплекса $\Delta$, а гр ебень - максимальная собственная грань фасеты. Двойственным графом $\Gamma(\Delta)$ комплекса $\Delta$ назьвается абстрактный граф̆, вершинами которого являются фасеты комплекса $\Delta$, а ребра соответствуют общим гребням фасет. Пусть $\sigma$ и $\tau$ - фасеты комплекса $\Delta$ такие, что $\sigma \cap \tau$ является гребнем обеих. Тогда существует единственная вершина $v(\sigma, \tau)$ фасеты $\sigma$, не принадлежащая $\tau$. Определим перспективное преобразование $[\sigma, \tau]: \sigma \rightarrow \tau$, положив

$$
v \mapsto \begin{cases}v(\tau, \sigma), & \text { если } v=v(\sigma, \tau), \\ v & \text { в противном случае. }\end{cases}
$$

Пусть $g=\left(\sigma_{0}, \sigma_{1}, \ldots, \sigma_{n}\right)$ - путь в $\Gamma(\Delta)$, т.е. $\sigma_{i}$ и $\sigma_{i+1}-$ фасеты, имеющие общий гребень, $i=0, \ldots, n-1$. Проективное преобразование, или, короче, проективность $[g]$ из $\sigma_{1}$ в $\sigma_{n}$ вдоль $g,-$ это результат последовательного выполнения перспективных преобразований:

$$
[g]=\left[\sigma_{0}, \sigma_{1}, \ldots, \sigma_{n}\right]=\left[\sigma_{0}, \sigma_{1}\right]\left[\sigma_{1}, \sigma_{2}\right] \cdots\left[\sigma_{n-1}, \sigma_{n}\right] .
$$


Отображение $[g]$ является биекцией из $\sigma_{0}$ в $\sigma_{n}$. Замкнутьй путь из $\sigma_{0}$ в $\sigma_{0}$ называется $n e m$ лей с началом в $\sigma_{0}$. Путь, получаемьй сочленением двух путей $g=\left(\sigma_{0}, \sigma_{1}, \ldots, \sigma_{n}\right)$ и $h=$ $\left(\sigma_{n}, \sigma_{n+1}, \ldots\right)$, обозначим через $g * h$. Очевидно, $[g * h]=[g][h]$.

Для данной грани $\sigma_{0}$ множество всех проективностей вдол петель с началом в $\sigma_{0}$ образует группу $\Pi\left(\Delta, \sigma_{0}\right)$ - группу проективностей комплекса $\Delta$ в фасете $\sigma_{0}$. Группа проективностей является подгруппой симметрической группы $\mathrm{Sym} \sigma_{0}$ - группы всех взаимно однозначных преобразований множества вершин фасеты $\sigma_{0}$. Если граф̆ $\Gamma(\Delta)$ связен (в этом случае комплекс $\Delta$ называется сильно связным $)$, то группы $\Pi\left(\Delta, \sigma_{0}\right)$, возникающие при различном выборе фасеты $\sigma_{0}$, изоморфны. Заметим также, что из связности дуального графа комплекса следует, что комплекс чист, т.е. все его фасеты имеют одинаковую размерность.

Следующий результат, доказанный в [6], является ключевым.

ТЕОРема 2. Для симплициального многообразия $\Delta$ его группа проективностей $\Gamma(\Delta)$ порождается совокупностью петель, соответствующих образующим фундаментальной группь, и петель вокруг граней коразмерности 2.

В частном случае, когда многообразие $\Delta$ односвязно, из теоремы 2 следует, что группа $\Pi\left(\Delta, \sigma_{0}\right)$ порождена транспозициями и, таким образом, изоморфна прямому произведению симметрических групп.

Для простого выпуклого многогранника $P$ әруппа проективностей $P$ определяется как группа проективностей $\partial P^{*}$. Грани коразмерности 2 комплекса $P^{*}$ соответствуют двумерным граням многогранника $P$. Следователњно, простой выпуклшй многогранник четен, если и только если его группа проективностей равна 0 . Очевидным образом можно начать построение правильной раскраски простого многогранника, стартуя с фасет вокруг любой его вершины. Такая раскраска продолжается на любую фасету. Продолжение однозначно и приводит к правилной раскраске всего множества фасет в том и только том случае, если группа проективностей многогранника обращается в 0 .

Я благодарен $\Phi$. Кёрнер и И. Изместьеву за стимулирующие обсуждения этой темы.

\section{СПИСОК ЛИТЕРАТУРЫ}

[1] M.W. Davis, T. Januszkiewicz // Duke Math. J. 1991. V. 62. № 2. P. 417-451. [2] В. М. Бухштабер, Т. Е. Панов // Труды МИАН. 1999. Т. 225. С. 96-131. [3] И. В. Изместьев // Матем. заметки. 2001. Т. 69. № 3. С. 375-382. [4] G. M. Ziegler. Lectures on Polytopes. Berlin: Springer-Verlag, 1998. [5] J. R. Munkres. Elements of Algebraic Topology. Redwood City, CA: Addison-Wesley, 1984. [6] M. Joswig. Projectivities in simplicial complexes and colourings of simple polytopes // Preprint. 